\title{
Efeitos a Curto e Longo Prazo de um Grupo de Desenvolvimento de Habilidades Sociais para Universitários
}

\author{
Vinicius Santos Ferreira ${ }^{1}$ \\ Maria Aparecida Oliveira \\ Luc Vandenberghe \\ Pontifícia Universidade Católica de Goiás
}

\begin{abstract}
RESUMO - Apesar da introdução na atuação comunitária de práticas baseadas em evidências, sabemos pouco sobre a eficácia das intervenções fora de contextos de pesquisa. A área dos treinamentos de habilidades sociais carece de estudos em settings de mundo real, com números suficientes de participantes e follow-up. Investigou-se, em 34 universitários, o efeito a curto e longo prazo de um grupo de treinamento de habilidades sociais, num contexto de mundo real. Escores de habilidades sociais (IHS) e ansiedade (IDATE) foram verificados antes, depois da intervenção e após um intervalo de follow up. Os escores em ambos os testes melhoraram do pré-teste para o pós-teste, e se mantiveram estáveis do pós-teste para o follow-up no intervalo de três meses a cinco anos.
\end{abstract}

Palavras-chave: treinamento de habilidades sociais, ansiedade, estudantes universitários, práticas baseadas em evidência

\section{Short and Long Term Effects of a Social Skills Development Group for University Students}

\begin{abstract}
In spite of the trend to implement evidence-based practices in community settings, we know little about the efficacy of interventions outside research contexts. There is a lack of empirical studies on the effects of social skills training in real-world contexts that include sufficient numbers of participants and follow up studies. The short and long-term effects of a social skills training of 34 college students were investigated in a real world setting. Social skills (IHS) and anxiety (STAI) scores were checked before and after the intervention and after a follow up interval. Both anxiety and social skills improved from the pre-test to post-test, and both remained stable from the post-test to follow-up after 3 months to 5 years.
\end{abstract}

Keywords: social skills training, anxiety, college students, evidence-based practice

O mercado de trabalho exige profissionais com capacidades de trabalhar em equipe, de liderança e de resolução de conflitos. Tais exigências ultrapassam o conhecimento formal especializado e exige que os sistemas educativos incluam um variado leque de competências (Primi, Santos, \& Vendramini, 2002), como novas formas de comunicação e de acesso à informação. A inserção no ambiente universitário também requer que o aluno seja autônomo, trabalhe em grupo, lide com críticas e defenda seus direitos (Gerk \& Cunha, 2006). Muitas das competências sociais exigidas pelo ambiente acadêmico relacionam-se com a habilidade de falar em público, como é o caso de expressar a opinião perante a turma, fazer perguntas, avaliações orais e apresentações de trabalho (Oliveira \& Duarte, 2004).

\section{Habilidades sociais: uma conceitualização}

Historicamente o campo das habilidades sociais foi construído de forma eclética, com maior influência por parte das abordagens cognitivas e comportamentais. Segundo Del

1 Endereço para Correspondência: Pontifícia Universidade Católica de Goiás, Primeira Avenida, Setor Leste Universitário, Goiânia - GO, CEP: 74605-220.E-mail: viniciusantosferreira@hotmail.com
Prette e Del Prette (2000), a origem desse campo deve-se a dois movimentos concomitantes: o do treinamento assertivo iniciado por Wolpe e Lazarus nos USA e o treinamento de habilidades sociais liderado por Argyle na Inglaterra. A categoria da assertividade foi absorvida dentro do universo mais amplo das habilidades sociais (Bolsoni-Silva et al., 2006; Del Prette \& Del Prette, 1996). Além desses, Bolsoni-Silva (2002) aponta outros modelos que contribuíram para o campo, como o modelo de aprendizagem social, de crenças irracionais, vulnerabilidade cognitiva, percepção social e da teoria dos papéis para citar alguns exemplos.

No Brasil, o desenvolvimento do campo conceitual das habilidades sociais foi tardio e tende a integrar diversos desenvolvimentos anteriores na literatura internacional. Del Prette e Del Prette (1996) incluem "todo e qualquer desempenho emitido em interações sociais" (p. 242) na categoria de habilidades sociais. Essas podem ser categorizadas nas funções: conceitual, que envolve um conjunto de suposições e teorizações que buscam explicar a relação interpessoal; e a descritiva, que se refere a classes de comportamentos relevantes para o desempenho em situações interpessoais. A avaliação do desempenho remete ao conceito de competência social. Em suma, as habilidades sociais aludem ao repertório de habilidades do indivíduo, enquanto a competência social se refere aos efeitos desses comportamentos sociais. 
Visto a amplitude da definição das habilidades sociais, Del Prette e Del Prette (2001) sugerem a divisão desse construto em grandes classes: habilidades de comunicação; civilidade; assertivas, de direito e cidadania; empáticas; de trabalho; e de expressão de sentimentos positivos. Déficits ou a falta de competência em qualquer um desses repertórios sociais, segundo Del Prette e Del Prette (2001), podem prejudicar as relações sociais, a qualidade de vida, ao passo que, pessoas socialmente competentes, em geral, apresentam melhores relações sociais, saúde e funcionamento psicológico. Essas demandas podem ser supridas por programas estruturados de treinamento de habilidades sociais, que são métodos de intervenção elaborados com essa finalidade (Del Prette \& Del Prette, 2011).

\section{Habilidades sociais, ansiedade e desempenho acadêmico}

Estudos têm apontado uma associação negativa entre ansiedade e habilidades sociais. Essa ideia não é nova, Salter (1931) - um dos pioneiros da terapia comportamental - baseado em diversos estudos de casos, propôs que a falta de habilidades sociais seria resultante de uma ansiedade condicionada. Bandeira, Quaglia, Bachetti, Ferreita e Souza (2005) se depararam com uma correlação negativa $(r=-0,55)$ entre assertividade e ansiedade. Em um contexto universitário, Furtado, Falcone e Clark (2003) encontraram que homens que tinham déficits em habilidades sociais apresentaram um nível de estresse mais alto. Iruarrizaga, Gómez-Segura, Criado, Zuazo e Sastre (1999) mostraram que um treinamento de habilidades sociais foi efetivo em diminuir o nível de ansiedade de seus participantes.

Habilidades sociais e ansiedade podem ter uma relação com o desempenho acadêmico. Um repertório empobrecido de habilidades sociais exigidas em situações acadêmicas pode prejudicar o desempenho acadêmico, ou gerar evasão (Oliveira \& Duarte, 2004). Em dois estudos empíricos (Gerk \& Cunha, 2006; Soares, Poubel, \& Mello, 2009) foram encontradas diversas correlações positivas entre fatores específicos de um inventário de habilidades sociais e alguns dos fatores pessoais e contextuais de um questionário de vivência acadêmica, embora essas correlações não tenham existido no escore total. A última relação, inversa, entre ansiedade e desempenho acadêmico foi exemplificada em outros dois estudos empíricos. Tanto Cassady e Johnson (2001) quanto Chapell et al. (2005) encontraram correlações negativas entre ansiedade e desempenho acadêmico.

Universitários que tenham uma ansiedade exacerbada podem se esquivar e/ou fugir de situações sociais. A fuga e esquiva podem prejudiar o desenvolvimento de um repertório de habilidades sociais adequado. Por sua vez, as dificuldades interpessoais poderiam suscitar mais ansiedade, gerando um círculo vicioso entre dificuldades interpessoais e ansiedade. Como ambos afetam o rendimento acadêmico dos estudantes, pode-se esperar que o desempenho seja comprometido progressivamente, à medida que o círculo vicioso se agrava.

\section{Estudos sobre treinamento de habilidades sociais no Brasil}

A área de treinamento de habilidades sociais aderiu ao movimento a favor da divulgação das práticas baseadas em evidências, que ganhou força em diferentes áreas da saúde (Del Prette \& Del Prette, 2011; Murta, 2011). Em revisões da literatura, Bolsoni-Silva et al. (2006) e Murta (2005) sinalizaram, respectivamente, 12 e 17 estudos empíricos sobre a efetividade de treinamentos de habilidades sociais no Brasil, esses abrangeram diversos campos de atuação profissional e diferentes populações. Os autores verificaram que a maioria desses trabalhos usou um delineamento pré-experimental, com apenas pré e pós-teste, desconsiderando a avaliação dos resultados em longo prazo (follow-up).

Dentre esses estudos de treinamentos de habilidades sociais são ainda mais escassos os que foram realizados em população acadêmica. Esses estudos seguiram diferentes contextos e objetivos. No contexto da formação de profissionais de psicologia, Del Prette, Del Prette e Barreto (1999), ao conduzir uma disciplina, compararam um grupo de alunos que receberam uma intervenção teórica contra outro grupo prático vivencial. Magalhães e Murta (2003) e Pacheco e Rangé (2006) também trabalharam com graduandos em psicologia, com os respectivos objetivos: desenvolver habilidades profissionais e aperfeiçoar habilidades sociais deficitárias. Estudantes universitários de diferentes cursos, com dificuldades interpessoais, foram alvos dos treinamentos de habilidades sociais avaliados por: Boas, Silveira e Bolsoni-Silva (2005); Bolsoni-Silva, Leme, Lima, Costa-Júnior e Correia (2009), incluindo estudantes recém graduados; e Falcone (1999), com um treinamento específico para o desenvolvimento de habilidades empáticas. Del Prette e Del Prette (2003a) treinaram habilidades sociais necessárias para o ambiente de trabalho em estudantes de exatas. Finalmente, Pontes e Souza (2011) analisaram a efetividade de um programa de treinamento de habilidades sociais para ajudar alunos de grupos sociais tradicionalmente desfavorecidos com dificuldade de adaptação ao ambiente acadêmico.

Os treinamentos de habilidades sociais se mostraram efetivos, apesar de carecerem, em geral, de maior rigor científico. Entre os estudos acima, três usaram um grupo controle (Del Prette et al., 1999; Falcone, 1999; Pacheco \& Rangé, 2007). Somente o estudo de Falcone (1999) apresentou follow-up, limitado a um mês. Apenas dois incluíram um número de participantes superior a 20 (Del Prette et al., 1999; Pacheco \& Rangé, 2007). Três deles não utilizaram inferências estatísticas na análise dos dados (Boas et al., 2005; Magalhães \& Murta, 2003; Pontes \& Souza, 2011). Essa falta de rigor científico deve ser alvo de futuras investigações.

\section{Contexto de mundo real}

O movimento para a disseminação de práticas baseadas em evidências favorece a prática do treinamento de habilidades sociais (Del Prette \& Del Prette, 2011; Murta, 2011). O rigor científico exigido para o estudo de efeitos terapêuticos pode entrar em conflito com as preocupações de um serviço 
assistencial (Tirado, Ortega, Dias, \& Martín, 1996/2005), fomentando a crítica que os resultados obtidos nas pesquisas controladas não garantam que o treino também funcione no mundo real. A diferença entre os contextos de pesquisa e assistencial deve ser levada a sério.

Os efeitos verificados em estudos empíricos rigorosamente controlados podem, com grande probabilidade, repetir-se caso sejam mantidas as mesmas condições em novos settings. Porém, na prática assistencial, raramente é possível seguir à risca os manuais. É preciso se adequar a demandas como: as necessidades dos usuários; condições limitadoras da prática; características da população, do ambiente e do momento; e o feedback dos participantes. Além disso, nos contextos assistenciais, muitas variáveis não podem ser controladas. Há problemas éticos na seleção aleatória dos participantes e na implementação de um grupo controle (Magalhães \& Murta, 2003). Essas modificações dos protocolos e falta de controle, em um contexto de "mundo real", podem afetar a efetividade das intervenções. As práticas baseadas em evidências precisam ser avaliadas em contextos de mundo real, onde o efeito da intervenção é verificada em consonância com as condições reais da implementação desses programas na comunidade.

Este estudo objetivou verificar o impacto de grupos de treinamento de habilidades sociais, a curto e longo prazo, sobre as habilidades sociais e a ansiedade de estudantes de uma instituição de ensino superior no Centro-Oeste brasileiro. A proposta foi de apresentar dados sobre um grupo de treino de habilidades sociais com caráter de "mundo real", isto é, um grupo que não foi montado com intenções de pesquisa, mas exemplifica a prática de treino de habilidades sociais num setting de serviço de atendimento aos estudantes.

O grupo existe desde 2004 e atende regularmente estudantes com déficits em habilidades sociais e ansiedade. Questionários pré e pós-intervenção eram aplicados nos participantes com a intenção de proporcionar um feedback aos mesmos. Especificamente para este estudo, antigos integrantes dos grupos foram convidados para a realização de uma medida de follow-up. Esses já haviam sido avaliados antes e depois da intervenção, três meses a cinco anos antes da realização desse estudo.

Os dados arquivados (pré e pós-teste), em conjunto com as medidas de follow-up permitiram avaliar, a curto e longo prazo, a implementação de um treinamento de habilidades sociais fora do contexto de pesquisa. Duas hipóteses foram propostas: a) esperou-se que do pré-teste para o pós-teste ocorresse uma melhora das habilidades sociais acompanhada de uma diminuição da ansiedade; e b) que esses efeitos se mantivessem em longo prazo.

\section{Método}

\section{Participantes}

Participaram do estudo 34 estudantes ou ex-estudantes de uma instituição de ensino de nível superior, privada, de uma cidade do Centro-Oeste brasileiro. Oito deles eram ho- mens e 26 mulheres, com a média de idade de 24,2 anos ( $\sigma$ $=8,18$ ). Todos participaram do grupo de desenvolvimento de habilidades para comunicação interpessoal e em público, que foi oferecido pelo setor de assistência estudantil da própria universidade. Os critérios para inclusão na amostra foram: ter participado de, no mínimo, $70 \%$ dos encontros e ter respondido os dois instrumentos de medida utilizados no pré-teste e no pós-teste, durante o período de 2004 a 2009.

Os participantes dos grupos eram estudantes regularmente matriculados em qualquer curso regular da instituição, de situação socioeconômica e idade variadas e que apresentassem algum nível de ansiedade e/ou dificuldade para enfrentar situações acadêmicas que exigiam exposição em público (fazer perguntas ou participar em sala, apresentar trabalhos acadêmicos, realizar avaliações escritas ou orais, interagir com pares ou autoridades) e que avaliassem que seu rendimento acadêmico estava sendo afetado por esses motivos.

\section{Descrição do Grupo}

O objetivo do grupo era promover a aquisição de habilidades sociais, emocionais e cognitivas que facilitassem a interação e integração ao ambiente acadêmico, bem como o manejo da ansiedade em situações de exposição em público, visando à qualificação da vida acadêmica. Normalmente, de dois a três grupos eram formados a cada semestre, desde 2004. Eram realizados 10 encontros, uma vez por semana, cada um com duração de duas horas e meia. Os grupos se iniciavam com 15 participantes e eram heterogêneos quanto aos escores de habilidades sociais e nível de ansiedade.

As intervenções foram feitas pela própria psicóloga coordenadora do programa, com a ajuda de diferentes estagiários que exerceram o papel de co-facilitadores. Os estagiários voluntários eram selecionados entre os alunos do curso de psicologia da instituição que estivessem cursando a partir do $5^{\circ}$ período. No primeiro período de atuação, realizavam atividades como participantes, observadores, apoio logístico, planejamento e avaliação das atividades. Um grupo de estudo e supervisão semanais complementaram o treinamento dos estagiários. A partir do segundo semestre, os estagiários atuavam como co-facilitadores.

O protocolo utilizado nos grupos de desenvolvimento de habilidades para comunicação interpessoal e em público foi desenvolvido a partir dos estudos de Beck (1997), Del Prette e Del Prette (2001), Echeburúa (1997), Hope e Heimberg (1999), Moscovici (1985) e White e Freeman (2003). Os procedimentos incluíam: reestruturação cognitiva, técnicas de manejo de ansiedade (respiração diafragmática e relaxamento muscular progressivo), treinamento de habilidades sociais (principalmente falar em público e treino assertivo), videofeedback, formulação de objetivos, exposição ao vivo, entre outras atividades. Uma característica deste trabalho foi a utilização do processo vivencial de aprendizagem, proposto por Moscovici (1985), a partir de dinâmicas de grupo para a aquisição e o fortalecimento dos repertórios. A Tabela 1 apresenta o protocolo de forma resumida. 
Tabela 1. Protocolo resumido do treinamento de habilidades sociais incluindo objetivos e estratégias utilizadas por sessão.

\begin{tabular}{|c|c|c|}
\hline Sessão & Objetivos das sessões & Estratégias utilizadas \\
\hline $1^{\mathrm{o}}$ & $\begin{array}{l}\text { Avaliação pré-intervenção. Apresentação do trabalho. } \\
\text { Aproximar e integrar o grupo. Levantar os objetivos de } \\
\text { cada participante. Elaboração do contrato. }\end{array}$ & $\begin{array}{l}\text { Aplicação dos inventários. } \\
\text { Exercícios e jogos para propiciar descontração, aproximação } \\
\text { e livre expressão. Exposição dialogada. }\end{array}$ \\
\hline $2^{o}$ & $\begin{array}{l}\text { Promover integração e afetividade no grupo. Compreender } \\
\text { a origem e função da ansiedade. Desenvolver o automoni- } \\
\text { toramento. Incentivar a experimentação comportamental. }\end{array}$ & $\begin{array}{l}\text { Exposição dialogada. Exercícios vivenciais com análise, } \\
\text { conceituação e conexão. Exercício de automonitoramento de } \\
\text { pensamentos, emoções e comportamentos. }\end{array}$ \\
\hline $3^{\circ}$ & $\begin{array}{l}\text { Reforçar integração do grupo. Continuar o treino de } \\
\text { automonitoramento. Aprender a Reestruturação Cognitiva } \\
\text { (RC). Reforçar a prática da experimentação comportamen- } \\
\text { tal. Aprendizagem de técnica para manejo da ansiedade. }\end{array}$ & $\begin{array}{l}\text { Exercícios vivenciais com análise, conceituação e conexão. } \\
\text { Definição de situações para experimentação comporta- } \\
\text { mental. Ensaio comportamental. Respiração Diafragmática } \\
\text { (RD). }\end{array}$ \\
\hline $4^{\circ}$ & $\begin{array}{l}\text { Aprender estratégias de enfrentamento de situações ansio- } \\
\text { gênicas. Continuar técnicas de manejo da ansiedade. }\end{array}$ & $\begin{array}{l}\text { Exercícios vivenciais com análise, conceituação e conexão. } \\
\text { Treinamento da RC, RD. Relaxamento muscular progressivo. }\end{array}$ \\
\hline $5^{\circ}$ & $\begin{array}{l}\text { Revisar exercícios de experimentação comportamental. } \\
\text { Treinar habilidades não verbais e paralinguísticas. Treinar, } \\
\text { oferecer e receber feedbacks. }\end{array}$ & $\begin{array}{l}\text { Exercícios vivenciais com análise, conceituação e conexão. } \\
\text { Exposição didática. Ensaio comportamental e reforçamento } \\
\text { social. }\end{array}$ \\
\hline $6^{\circ}$ & $\begin{array}{l}\text { Revisar exercícios de experimentação comportamental. } \\
\text { Treinar, oferecer e receber feedback. Treinar habilidades } \\
\text { não verbais e paralinguísticas. Treinar a RC. }\end{array}$ & $\begin{array}{l}\text { Exercícios vivenciais com análise, conceituação e conexão; } \\
\text { Ensaio comportamental e reforçamento social; }\end{array}$ \\
\hline $7^{\circ}$ & $\begin{array}{l}\text { Treinar habilidades não verbais e paralinguísticas. Iniciar } \\
\text { treinamento assertivo. }\end{array}$ & $\begin{array}{l}\text { Exercícios vivenciais com análise, conceituação e conexão; } \\
\text { Exposição dialogada. Ensaio comportamental e modelação. }\end{array}$ \\
\hline $8^{\circ}$ & Treinar habilidades aprendidas. Continuar treino assertivo. & $\begin{array}{l}\text { Exercícios vivenciais com análise, conceituação e conexão. } \\
\text { Ensaio comportamental, reforçamento social e modelação. }\end{array}$ \\
\hline $9^{\circ}$ & $\begin{array}{l}\text { Treino de habilidades aprendidas com uso de palco e de } \\
\text { recursos audiovisuais. }\end{array}$ & $\begin{array}{l}\text { Apresentação com uso de recursos audiovisuais. Exposição } \\
\text { dialogada, modelação, reforçamento social; feedback. }\end{array}$ \\
\hline $10^{\mathrm{o}}$ & $\begin{array}{l}\text { Avaliação pós-intervenção. Avaliação do programa e } \\
\text { encerramento. }\end{array}$ & $\begin{array}{l}\text { Videofeedback. Aplicação dos inventários. Avaliação da } \\
\text { aprendizagem. }\end{array}$ \\
\hline
\end{tabular}

\section{Instrumentos}

O IHS (Inventário de Habilidades Sociais), formulado e validado para população brasileira por Del Prette e Del Prette (2001), é um instrumento de fácil aplicação com o objetivo de caracterizar o desempenho social, de adultos e jovens, em diferentes situações (trabalho, escola, família e cotidiano). O Alfa de Cronbach, $(0,75)$ indica uma consistência interna satisfatória. $\mathrm{O}$ inventário é composto de 38 afirmativas que apresentam variadas situações, incluindo reações emocionais e comportamentos. O respondente deve avaliar a frequência com que age ou se sente tal como descrito no item.

O nível de ansiedade de cada participante foi avaliado por meio do IDATE, validado para população brasileira (Spielberger, Biaggio, \& Natalício, 1979), que é subdividido em Inventário de Ansiedade A-Traço e Inventário de Ansiedade A-Estado. A Escala A-Traço contém 20 afirmações e requer que os indivíduos indiquem como geralmente se sentem. Refere-se a diferenças individuais na tendência de reagir a situações percebidas como ameaçadoras com elevação na intensidade da ansiedade. A consistência interna, medida pelo Alfa de Cronbach é de 0,93 para homens e 0,87 para mulheres.

\section{Procedimentos}

Primeiro foi realizado um levantamento do material arquivado de todos os grupos realizados no período de 2004 a 2009. Foram identificados: nome, endereço, telefone e $e$ -mail de 131 ex-participantes dos grupos que atendiam aos critérios da pesquisa. Em seguida, foi elaborada uma lista com os dados dos participantes. Um convite via $e$-mail foi enviado a todos os sujeitos selecionados para pesquisa, agendando local e horário. No caso dos que não responderam ao 
e-mail ou não compareceram, um novo contato foi efetuado por telefone, no qual foram agendados horários, de acordo com a disponibilidade dos participantes. A maior causa de não participação no estudo deveu-se à desatualização dos dados de contato dos ex-participantes dos grupos, inferidas pela falta de resposta aos e-mails e telefonemas. Outras causas importantes foram a mudança de cidade e a falta de tempo. Após se apresentarem, os participantes responderam a uma avaliação de follow-up contendo o IHS e o IDATE. Essa avaliação foi realizada de forma individual ou em grupos de dois a quatro participantes, sob supervisão do pesquisador responsável.

Todos os participantes que compareceram, leram e assinaram um termo de consentimento livre e esclarecido explicando a pesquisa, seus objetivos e procedimentos. Foi garantido o sigilo das informações prestadas, e explicitado que as informações seriam analisadas estatisticamente, sendo impossível identificar os participantes. A possibilidade de se retirar a qualquer momento, sem qualquer forma de prejuízo, foi garantida. Os parâmetros éticos para pesquisa com seres humanos foram assegurados.

\section{Análise dos Dados}

Primeiro, todos os participantes foram comparados com eles mesmos nas três avaliações: pré-teste, pós-teste e follow-up. O período de follow-up variou de três meses a cinco anos. Em um segundo momento, verificou-se a influência desses diferentes períodos dividindo os participantes em três subgrupos. O "grupo 1" com follow-up de três meses a um ano ( $=10)$; "grupo 2" com follow-up de um ano e seis meses a três anos $(\mathrm{N}=10)$; e "grupo 3" com follow-up de três anos e seis meses a cinco anos $(\mathrm{N}=14)$. Para as análises, foram realizadas análises de variância (ANOVA) de medidas repetidas para primeira análise e uma ANOVA de amostras independentes para a segunda. Para uma análise post hoc, o teste $t$ de medidas repetidas foi usado com o objetivo de desmembrar o efeito da intervenção, a curto e longo prazo, em pares de avaliações pré-teste/pós-teste, pré-teste/follow-up e pós-teste/follow-up.

Para se obter uma verificação individualizada da eficácia e da efetividade da intervenção, utilizou-se o método JT, desenvolvido inicialmente por Jacobson e Truax (1991). Esse método propõe a avaliação de duas medidas: o índice de mudança confiável, que mede o erro de medição, relacionado à validade interna, considerando a confiabilidade dos instrumentos de medida e seus erros padrões associados; e a significância clínica, relacionado à validade externa, comparando os sujeitos com grandes amostras funcionais e/ou disfuncionais previamente conhecidas, o que permite inferir a importância prática das mudanças observadas após a intervenção (Del Prette \& Del Prette, 2008). Segundo os critérios de operacionalização da medida de significância clínica, propostos por Jacobson e Truax (1991), utilizou-se neste estudo o critério "B", visto que se dispõe de dados normativos apenas da população funcional. Esses dados para o IHS e o IDATE foram retirados, respectivamente, dos trabalhos de Del Prette e Del Prette (2001) e de Gama, Moura, Araújo e Teixeira-Silva (2008).
A correlação entre os escores de habilidades sociais e o nível de ansiedade foi verificada com o coeficiente de correlação de Pearson. Todas as análises foram realizadas a partir do escore geral bruto do IHS e do escore bruto do IDATE. As análises foram feitas sempre para o Inventário de Ansiedade A-Traço. Para efetuar as análises, os programas utilizados foram: o Pacote Estatístico para as Ciências Sociais (SPSS), versão 18, e o Psicoinfo Análises Informatizadas [software online]. O nível de significância considerado em todos os casos foi de 0,05 .

\section{Resultados}

A Tabela 2 apresenta os dados descritivos - a medida mínima e máxima, a média e o desvio padrão - do IHS e do IDATE, para as três etapas estudadas: pré-teste, pós-teste e follow-up.

Para verificar se o treinamento de habilidades sociais afetou o escore de habilidades sociais e o nível de ansiedade foram realizadas duas ANOVAs de medidas repetidas: uma para o IHS $F(2,31)=30,8, \mathrm{p}<0,000$ e outra para o IDATE $F(2,31)=28,3, \mathrm{p}<0,000$. Os resultados indicaram diferenças estatisticamente significativas entre o pré-teste, o pós-teste e o follow-up, nas duas medidas, IHS e IDATE.

Dada essa diferença, com o intuito de especificar o efeito da intervenção proposta, a partir do teste $t$ de medidas repetidas, foram comparadas separadamente as três possibilidades de pares de avaliações, pré-teste/pós-teste, pré-teste/follow-up e pós-teste/follow-up, para o IHS e o IDATE (ver Tabela $3)$. Diferenças estatisticamente significativas foram encontradas para o IHS do pré-teste para o pós-teste e do pré-teste para o follow-up e não foi identificada do pós-teste para o follow-up, o mesmo repetiu-se no IDATE. Significa que, no caso do IHS, os escores de habilidades sociais aumentaram do pré-teste para o pós-teste e se mantiveram estáveis do pós-teste para o follow-up. O mesmo ocorreu com o IDATE, porém se trata do nível de ansiedade e não dos escores de habilidades sociais. Assim verificou-se uma diminuição e não um aumento.

Como no trabalho houve diferentes períodos de follow-up, variando de três meses a cinco anos, os participantes da pesquisa foram separados em grupo 1, 2 e 3, como descrito na sessão análise dos dados. Esse procedimento possibilitou que fosse investigada a relação de tempo de follow-up com as variáveis dependentes. Tanto para o IHS quanto para o IDATE, foram realizados ANOVAs comparando os três grupos na avaliação de follow-up. Os resultados foram: $F(2$, $31)=2,975, p=0,066(\mathrm{IHS})$; e $F(2,31)=0,813, p=0,453$, (IDATE). Os testes indicaram que não existem diferenças estatisticamente significativas entre os três grupos, para nenhuma variável dependente. Como os grupos 1, 2 e 3 não se diferiram entre si, as outras análises foram efetuadas com a amostra total do estudo.

Com a utilização do método JT, foram analisados o índice de mudança confiável e a significância clínica da intervenção proposta. As análises foram feitas comparando o pré-teste com o pós-teste, no IHS e no IDATE. As avaliações de follow-up não foram incluídas, visto que os resultados se mantiveram semelhantes ao do pós-teste. Os resultados 
Tabela 2. Média e Desvio Padrão do IHS e IDATE no pré-teste, pós-teste e follow up.

\begin{tabular}{|c|c|c|c|c|c|}
\hline Inventários & $\mathrm{N}$ & Escore Mínimo & Escore Máximo & Escore Médio & Desvio Padrão \\
\hline IHS/Pré-teste & 34 & 29 & 118 & 74,21 & 19,64 \\
\hline IHS/Pós-teste & 34 & 58 & 128 & 98,97 & 17,61 \\
\hline IHS/Folow up & 34 & 53 & 133 & 97,74 & 18,35 \\
\hline IDATE/Pré-teste & 34 & 41 & 72 & 53,15 & 8,62 \\
\hline IDATE/Pós-teste & 34 & 28 & 64 & 41,74 & 9,02 \\
\hline IDATE/Folow up & 34 & 21 & 57 & 40,74 & 8,03 \\
\hline
\end{tabular}

foram apresentados em um formato gráfico para facilitar a interpretação. A Figura 1 compara o pré-teste e o pós-teste para o IHS, enquanto a Figura 2 repete o mesmo procedimento para o IDATE.

Cada um dos pontos no gráfico representa um participante. A linha central que corta o gráfico na diagonal é chamada de bissetriz, as linhas paralelas a ela representam seu erro padrão, a bissetriz representa o índice de mudança confiável. Os participantes que se encontram acima dessa linha tiveram uma mudança confiável do pré-teste para o pós-teste, enquanto que os que estão abaixo dela, pioraram após a intervenção. Não se pode afirmar com segurança se os participantes que se encontram entre as linhas do erro padrão melhoraram ou pioraram.

A significância clínica é representada no gráfico pelas linhas centrais, vertical e horizontal, as linhas paralelas a elas indicam seu erro padrão. Essas linhas dividem os gráficos em quatro quadrantes (A) superior esquerdo, (B) superior direito, (C) inferior esquerdo, e (D) inferior direito. O quadrante A inclui os participantes que necessitavam de intervenção e obtiveram uma melhora no pós-teste. O quadrante B mostra os participantes que estavam em uma faixa não clínica da população (escores altos de habilidades sociais e baixos de ansiedade) e assim permaneceram. O quadrante $\mathrm{C}$, ao contrário do $\mathrm{B}$, mostra os participantes que estavam dentro da faixa clínica da população e se mantiveram da mesma forma. $\mathrm{O}$ quadrante $\mathrm{D}$ contém os participantes que estavam em uma faixa não clínica da população e mudaram para a faixa clínica, ou seja, os que pioraram após a intervenção.

Podemos verificar na Figura 1 que 67,6\% dos participantes tiveram um aumento confiável no escore de habilidades sociais após a intervenção. Apenas 5,9\% tiveram uma diminuição desse escore e 26,5\%, uma ausência de mudança. Quanto à significância clínica da intervenção, apenas oito participantes tinham um escore no pré-teste que os colocava em uma situação clínica, ou seja, aqueles que se encontram à esquerda da linha vertical. Desses, seis migraram para o quadrante $\mathrm{A}$, sendo que cinco $(62,5 \%)$ obtiveram uma melhora clínica significativa no pós-teste somada a uma mudança confiável no escore de habilidades sociais. O contrário, uma deterioração das habilidades sociais, não ocorreu em nenhum caso, o que pode ser verificado pela ausência de participantes no quadrante D. A maior parte dos participantes $(61,8 \%)$ ficou no quadrante $B$, eles já estavam em uma população não clínica e assim permaneceram, e mesmo entre esses, a maioria mostrou uma melhora confiável, por estar acima da margem de erro da bissetriz.

A Figura 2 está invertida, pois uma diminuição do nível de ansiedade significa uma melhora clínica. Analisando o gráfico observam-se mudanças confiáveis nos níveis de ansiedade positivas e negativas, respectivamente, em $58,8 \%$ e $2,9 \%$ dos participantes. Nos outros $38,2 \%$ dos sujeitos houve uma ausência de mudança confiável; $62,5 \%$ dos participantes que no pré-teste apresentaram níveis clínicos de ansiedade mudaram para índices funcionais de ansiedade no pós-teste (quadrante A). No pós-teste, apenas dois participantes se encontraram em uma faixa clínica da população, um no quadrante $\mathrm{C}$ e outro no quadrante $\mathrm{D}$, nenhum deles apresentou mudanças confiáveis. Como na análise dos escores de habilidades sociais, 70,6\% dos sujeitos se encontrou no quadrante B, desses 58,3\% melhoraram ainda mais após a intervenção, contra apenas um caso de piora confiável.

Por último, verificou-se a relação entre habilidades sociais e ansiedade. Foram encontradas correlações negativas significativas entre os escores de habilidades sociais e o nível de ansiedade, no pré-teste, de $r=-0,521, p=0,002$; no pós-teste de $r=-0,464, p=0,006$; e no follow-up de $r=-0,595, p<0,000$.

Tabela 3. Resultado do teste $t$ nos pares indicados no IHS e IDATE.

\begin{tabular}{|c|c|c|c|c|c|}
\hline Pares IHS & $t(33)$ & $p$ & Pares IDATE & $t(33)$ & $p$ \\
\hline $\begin{array}{l}\text { IHS/Pré-teste x } \\
\text { IHS/Pós-teste }\end{array}$ & $-7,205$ & 0,000 & $\begin{array}{l}\text { IDATE/Pré-teste x IDATE/ } \\
\text { Pós-teste }\end{array}$ & 6,662 & 0,000 \\
\hline $\begin{array}{l}\text { IHS/Pré-teste } \mathrm{x} \\
\text { IHS/Folow up }\end{array}$ & $-5,626$ & 0,000 & $\begin{array}{l}\text { IDATE/Pré-teste x IDATE/ } \\
\text { Folow up }\end{array}$ & 6,018 & 0,000 \\
\hline $\begin{array}{l}\text { IHS/Pó-teste x } \\
\text { IHS/Folow up }\end{array}$ & 0,419 & 0,678 & $\begin{array}{l}\text { IDATE/Pós-teste x IDATE/ } \\
\text { Folow up }\end{array}$ & 0,587 & 0,561 \\
\hline
\end{tabular}




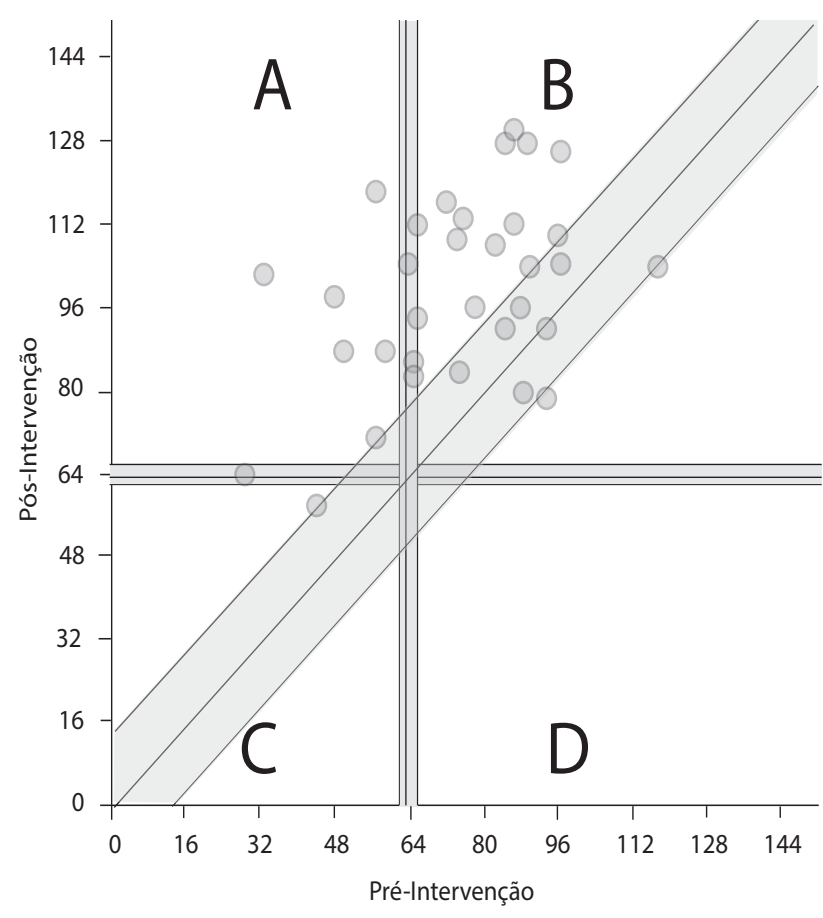

Figura 1. Resultados da confiabilidade da mudança e da significância clínica nos escores brutos do IHS, para cada participante, ao se comparar o pré-teste com o pós-teste.

\section{Discussão}

A maioria dos participantes deste estudo beneficiou-se do programa de treinamento de habilidades sociais e os benefícios obtidos, um aumento dos escores de habilidades sociais e uma diminuição do nível do traço de ansiedade foram mantidos em longo prazo. No que diz respeito às habilidades sociais, o método JT (Figura 1) deixou claro que apenas dois participantes tiveram uma piora confiável nos escores de habilidades sociais e em nenhum dos casos houve uma piora clínica, ou seja, nenhum participante se encontrou no quadrante D. Como se trata de uma população comunitária, sem critério clínico de seleção, muitos já iniciaram os grupos com escores normais de habilidades sociais, por isso muitos participantes encontram-se no quadrante B. Mesmo assim, pode-se observar que a maioria aumentou seus escores do pré-teste para o pós-teste. A análise do método JT para os níveis de ansiedade-traço (Figura 2) apontou um resultado semelhante ao da Figura 1, de habilidades sociais. Poucos participantes se encontraram nos quadrantes C e D, enquanto a maior parte estava nos quadrantes A e B. Isso significa que ao final da intervenção, uma grande parcela dos participantes encontrou-se em uma faixa funcional da população, além de apresentar uma redução confiável nos níveis de ansiedade.

As melhoras nos escores habilidades sociais e no nível de ansiedade se mantiveram em longo prazo, com os períodos de follow-up variando entre os participantes de três meses até cinco anos. Não ocorreram oscilações significativas desses resultados ao longo desses cinco anos do estudo, o que indica uma estabilidade dos resultados desse programa de treinamento de habilidades sociais por um longo período, sem que houvesse uma deterioração das melhoras após a intervenção.

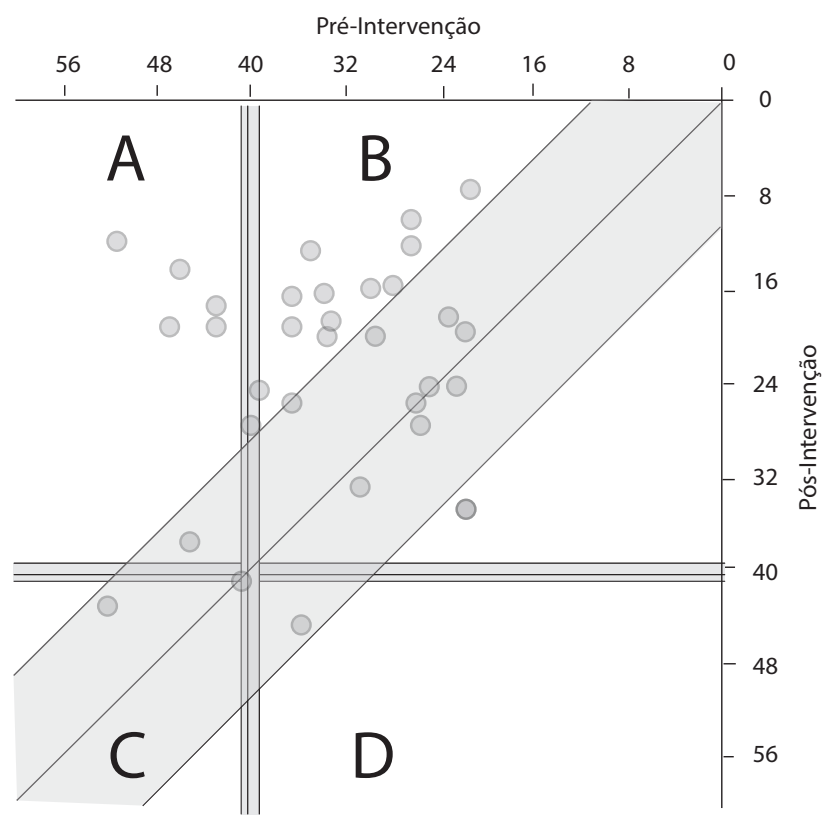

Figura 2. Resultados da confiabilidade da mudança e da significância clínica nos escores brutos do IDATE, para cada participante, ao se comparar o pré-teste com o pós-teste.

As correlações, entre habilidades sociais e ansiedade, encontradas em todas as fases de avaliação deste estudo reforçam os achados de Bandeira et al. (2005) que encontraram uma correlação entre déficit de repertório assertivo e alto nível de ansiedade. Baseado nessas correlações, pode-se supor que o treinamento de habilidades sociais contribuiu para a diminuição do nível de ansiedade, o que endossa os resultados dos estudos de Iruarrizaga et al. (1999), que também demonstraram que um treinamento de habilidades sociais foi efetivo em diminuir o nível de ansiedade.

Grupos de treinamento de habilidades sociais são comuns em settings comunitários (Murta, 2011), sendo uma prática tão divulgada merecem mais estudos do que já existem. A validação desse tipo de programa, como sendo uma prática baseada em evidência, apoia uma forma de trabalhar já amplamente instituída. No ambiente acadêmico, a falta de estudos empíricos acentua-se. Del Prette e Del Prette (2001) sugerem que uma melhora nas habilidades sociais pode contribuir para melhorar as relações sociais, a saúde e o funcionamento psicológico dos participantes. Além disso, uma pesquisa empírica mostrou que os acadêmicos com escores de habilidades sociais mais elevados são capazes de uma maior variedade de comportamentos interpessoais e flexibilidade nas interações com outras pessoas, enquanto que os com escores baixos tendem a usar de forma rígida sempre os mesmos tipos de comportamento nas suas interações (Couto, Vandenberghe, Tavares, \& Silva, 2012).

Para Del Prette e Del Prette (2003b), a competência social deveria ser uma das capacidades incluídas no escopo da formação universitária, visto que essas capacidades seriam necessárias nas relações acadêmicas e para as futuras relações de trabalho, as quais os estudantes são preparados. Segundo os autores, essa capacidade faz parte de um currículo oculto, 
sendo um subproduto do ensino geral. Uma inclusão formal e planejada dessas atividades poderia ser mais efetiva para o desenvolvimento dessa capacidade. Além desses ganhos sociais diretos, um treinamento de habilidades sociais, em população universitária, pode também ser benéfico para melhorar o desempenho acadêmico, vista a relação entre um desempenho socialmente competente e um baixo nível de ansiedade com um aumento no rendimento acadêmico (Cassady \& Johnson, 2001; Chapell et al., 2005; Gerk \& Cunha, 2006; Soares, et al., 2009). Outro beneficio importante seria a diminuição da evasão universitária considerando que Bardagi e Hutz (2012) demonstram, em um estudo qualitativo, que as relações sociais com outros alunos e professores são um fator importante para a adesão e conclusão do curso, enquanto relações insatisfatórias podem levar à evasão.

\section{Contribuições e limitações do estudo}

Duas contribuições importantes deste estudo foram um extenso follow-up e um grande número de participantes, enquanto as limitações encontradas foram a ausência de grupo controle e da seleção aleatória de participantes, o que caracteriza o estudo como pré-experimental.

Um estudo de follow-up com duração de até cinco anos representa uma contribuição inédita para a literatura brasileira. Encontrou-se somente um estudo anterior nesse contexto que utiliza uma avaliação de follow-up, sendo que o primeiro (Falcone, 1999) teve um enfoque diferenciado, estudando especificamente a empatia e teve intervalo de follow-up de apenas 30 dias. $\mathrm{O}$ intervalo de três meses a cinco anos do presente estudo permitiu uma verificação detalhada do efeito da passagem do tempo sobre os resultados do grupo. Apenas dois estudos encontrados (Del Prette et al., 1999; Pacheco \& Rangé, 2007) apresentaram uma amostra superior a 20 sujeitos. O tamanho da amostra do presente estudo, contando com 34 participantes, é ainda mais importante, visto que se trata de um grupo que funciona em um contexto comunitário sem intenção de pesquisa, o que acarreta numa maior dificuldade de acesso a participantes.

Em contrapartida, o trabalho sofreu dos típicos problemas encontrados na pesquisa em settings comunitários fora do contexto de pesquisa científica. Além da ausência de grupo controle e seleção aleatória dos participantes, do total de sujeitos convidados a participar do estudo, apenas $26 \%$ compareceram e não houve um critério clínico para a admissão nos grupos, como é recomendado para grupos de tratamento (Bolsoni-Silva et al., 2009). Outro problema foi que os dados deste estudo foram obtidos por meio de autorrelato. Não temos certeza de se os mesmos resultados seriam obtidos com outros métodos de pesquisa, tais como observação direta, com contagem de diferentes categorias de comportamentos, em ambientes naturais ou testes de desempenho em role-play. Uma avaliação dos componentes cognitivos trabalhados durante a intervenção também foi uma limitação deste estudo.

Para a análise dos resultados, foi utilizado apenas o escore total do IHS, e não os fatores específicos que o inventário permite. A vantagem dessa opção foi possibilitar uma visão geral do nível de habilidades sociais de cada participante, porém não permitiu analisar os componentes do IHS.

\section{Conclusão}

Os resultados mostram que a participação no grupo levou a um aumento dos escores de habilidades sociais e uma diminuição dos níveis de ansiedade. Os efeitos foram estatisticamente significativos a curto, médio e longo prazo. Portanto, as duas hipóteses foram confirmadas, indicando a eficácia e efetividade da implementação, em um setting comunitário sem intuito de pesquisa, de um treinamento de habilidades sociais. Logo, a prática de treinamento de habilidades sociais seguindo os parâmetros descritos nesta pesquisa, pode ser considerada uma prática baseada em evidência.

\section{Referências}

Bandeira, M., Quaglia, M. A. C., Bachetti, L. S., Ferreita, T. L., \& Souza, G. G. (2005). Comportamento assertivo e sua relação com ansiedade, locus de controle e auto-estima em estudantes universitários. Estudos em Psicologia (Campinas), 22(2), 111-121.

Bardagi, M. P., \& Hutz, C. S. (2012). Rotina acadêmica e relação com colegas e professores: impacto na evasão universitária. Psico, 43(2), 174-184.

Beck, J. S. (1997). Terapia cognitiva: teoria e prática. Porto Alegre: Artmed.

Boas, A. C. V. V., Silveira, F. F., \& Bolsoni-Silva, A. T. (2005). Descrição de efeitos de um procedimento de intervenção em grupo com universitários: um estudo piloto. Interação em Psicologia, 9(2), 321-330.

Bolsoni-Silva, A. T. (2002). Habilidades sociais: breve análise da teoria e da prática à luz da análise do comportamento. Interação em Psicologia, 6(2), 233-242.

Bolsoni-Silva, A. T., Del Prette, Z. A. T., Del Prette, G., Montagner, A. R., Bandeira, M., \& Del Prette, A. (2006). Aárea das habilidades sociais no Brasil: uma análise dos estudos publicados em periódicos. In M. Bandeira, Z. A. P. Del Prette, \& A. Del Prette, (Eds.), Estudos sobre habilidades sociais e relacionamento interpessoal (pp. 17-45). São Paulo: Casa do Psicólogo.

Bolsoni-Silva, A. T., Leme, V. B. R., Lima, A. M. A., Costa-Júnior, F. M., \& Correia M. R. G. (2009). Avaliação de um treinamento de habilidades sociais (THS) com universitários e recémformados. Interação em Psicologia, 13(2), 241-251.

Cassady, J. C., \& Johnson, R. E. (2001). Cognitive test anxiety and academic performance. Contemporary Educational Psychology, 27, 270-295.

Chapell, M. S., Blanding, B., Silverstein M. E., Takahashi, M., Newman, B., Gubi, A., \& McCann, N. (2005). Test anxiety and academic performance in undergraduate and graduate students. Journal of Educational Psychology, 97(2), 268-274.

Couto, G., Vandenberghe, L., Tavares, W. M., \& Silva, R. L. F. C. (2012). Interações e habilidades sociais entre universitários: um estudo correlacional. Estudos de Psicologia (Campinas), 29, 667-677. 
Del Prette, A., \& Del Prette, Z. A. P. (2001). Psicologia das relações interpessoais: vivências para o trabalho em grupo. Petrópolis: Vozes.

Del Prette, A., \& Del Prette, Z. A. P. (2003a). No contexto da travessia para o ambiente de trabalho: treinamento em habilidades sociais com universitários. Estudos de Psicologia (Natal), 8(3), 413-420.

Del Prette, A., \& Del Prette, Z. A. P. (2011). Enfoques e modelos do treinamento de habilidades sociais. In A. Del Prette, \& Z. A. P. (Eds.), Habilidades sociais: intervenções efetivas em grupo (pp. 19-56). São Paulo: Casa do Psicólogo.

Del Prette, A., Del Prette, Z. A. P., \& Barreto, M. C. M. (1999). Habilidades sociales en la formación profesional del psicólogo: análisis de un programa de intervención. Psicología Conductual, 7(1), 27-47.

Del Prette, Z. A. P., \& Del Prette, A. (1996). Habilidades sociais: uma área em desenvolvimento. Psicologia: Reflexão e Crítica, 9(2), 287-389.

Del Prette, Z. A. P., \& Del Prette, A. (2000). Treinamento de habilidades sociais: panorama geral da área. In V. G. Haase, R. Rothe-Neves, C. Kappçes, M. L. M. Teodoro, \& G. M. O. Wood (Eds.), Psicologia do desenvolvimento: contribuições interdisciplinares (pp. 249-264). Belo Horizonte: Health.

Del Prette, Z. A. P., \& Del Prette, A. (2001). Inventário de Habilidades Sociais (IHS-Del-Prette): manual de apuração e interpretação. São Paulo: Casa do Psicólogo.

Del Prette, Z. A. P., \& Del Prette, A. (2003b). Desenvolvimento interpessoal: uma questão pendente no ensino universitário. In E. Mercuri \& S. Polydoro (Eds.), Estudante universitário: características e experiências de formação (pp. 105-128). Taubaté: Cabral.

Del Prette, Z. A. P., \& Del Prette, A. (2008). Significância clínica e mudança confiável na avaliação de intervenções psicológicas. Psicologia: Teoria e Pesquisa, 24(4), 497-505.

Echeburúa, E. (1997). Vencendo a timidez. São Paulo: Mandarim.

Falcone, E. (1999). Avaliação de um programa de treinamento de empatia com universitários. Revista Brasileira de Terapia Comportamental e Cognitiva, 1(1), 23-32.

Furtado E. S., Falcone, E. M. O., \& Clark, C. (2003). Avaliação do estresse e das habilidades sociais na experiência acadêmica de estudantes de medicina de uma universidade do Rio de Janeiro. Interação em Psicologia, 7(2), 43-51.

Gama, M. M. A., Moura, G. S., Araújo, R. F., \& Teixeira-Silva, F. (2008). Ansiedade-traço de estudantes universitários de Aracajú (SE). Revista de Psiquiatria do Rio Grande do Sul, 30(1), 19-24.

Gerk, E., \& Cunha, S. M. (2006). As habilidades sociais na adaptação de estudantes ao ensino superior. In M. Bandeira, Z. A. P. Del Prette \& A. Del Prette (Eds.), Estudos sobre habilidades sociais e relacionamento interpessoal (pp. 199216). São Paulo: Casa do Psicólogo.

Hope, D. A., \& Heimberg R. G. (1999). Fobia social e ansiedade social. In D. H. Barlow (Ed.), Manual clínico dos transtornos psicológicos (pp. 119-160) (2 ${ }^{\mathrm{a}}$ ed.). Porto Alegre: Artmed.

Iruarrizaga, I., Gómez-Segura, J., Criado, T., Zuazo, M., \& Sastre, E. (1999). Reducción de la ansiedad a través del entrenamiento en habilidades sociales. Revista Electrónica de Motivación y Emoción, 2(1). Retrieved from http://reme.uji.es/articulos/ airuai462031198/texto.html
Jacobson, N. S., \& Truax, P. (1991). Clinical significance: a statistical approach to defining meaningful change in psychotherapy research. Journal of Consulting and Clinical Psychology, 59(1), 12-19.

Magalhães, P. P., \& Murta, S. G. (2003). Treinamento de habilidades sociais em estudantes de psicologia: um estudo pré-experimental. Temas de Psicologia da SBP, 11(1), 28-37.

Moscovici, F. (1985). Desenvolvimento interpessoal: treinamento em grupo ( $3^{\mathrm{a}}$ ed.). Rio de Janeiro: José Olympio.

Murta, S. G. (2005). Aplicações do treinamento em habilidades sociais: análise da produção nacional. Psicologia: Reflexão e Crítica, 18(2), 283-291.

Murta, S. G. (2011). Aproximando a ciência e a comunidade: a difusão de programas de habilidades sociais baseados em evidência. In A. Del Prette, \& Z. A. P. (Eds.), Habilidades sociais: intervenções efetivas em grupo (pp. 83-114). São Paulo: Casa do Psicólogo.

Oliveira, M. A., \& Duarte, A. M. M. (2004). Controle de respostas de ansiedade em universitários em situações de exposição oral. Revista Brasileira de Terapia Comportamental e Cognitiva, 6(2), 183-199.

Pacheco, P., \& Rangé, B. P. (2006). Desenvolvimento de habilidades sociais em graduandos de psicologia. In M. Bandeira, Z. A. P. Del Prette, \& A. Del Prette, (Eds.), Estudos sobre habilidades sociais e relacionamento interpessoal (pp. 199-216). São Paulo: Casa do Psicólogo.

Pontes, M. G. F. C., \& Souza, M. A. (2011). Treino de habilidades sociais em estudantes da Universidade Federal do Recôncavo da Bahia: uma possibilidade de atuação do psicólogo escolar educacional no ensino superior. Entrelaçando, 2(4), 116-126.

Primi, R., Santos, A. A. A., \& Vendramini, C. M. (2002). Habilidades básicas e desempenho acadêmico em universitários ingressantes. Estudos de Psicologia, 7(1), 47-55.

Salter, A. (1931). Conditioned reflex therapy: the direct approach to the reconstruction of personality. Londres: George Allan \& Unwin.

Soares, A. B., Poubel, L. M., \& Mello, T. V. S. (2009). Habilidades sociais e adaptação acadêmica: um estudo comparativo em instituições de ensino público e privado. Aletheia, 29, 27-42.

Spielberger, C., Biaggio, A. M. B., \& Natalício, R. E. (1979). Inventário de ansiedade traço-estado (IDATE): manual de psicologia aplicada. Rio de Janeiro: CEPA.

Tirado, J. L. A., Ortega, S. L., Días, V. A. H., \& Martín, F. D. F. (2005). Terapia breve en estudiantes universitarios con problemas de rendimiento académico y ansiedad: eficacia del modelo "la cartuja". International Journal of Clinical and Health Psychology, 5(3), 589-608. Tradução organizada por M. D. Claudino. São Paulo: Santos. (Trabalho original publicado em 1996)

White. J. R. E, \& Freeman, A. S. (2003). Terapia cognitivocomportamental em grupo para populações e problemas específicos. São Paulo: Rocca.

Recebido em 14.05.2012

Primeira decisão editorial em 09.06.2013

Versão final em 19.07.2013

Aceito em 07.08.2013 